\title{
Molecular characterization and immune modulation properties of Clonorchis sinensis-derived RNASET2
}

Yanquan $\mathrm{Xu}^{1,2}$, Wenjun Chen ${ }^{1,2}$, Meng Bian ${ }^{1,2}$, Xiaoyun Wang ${ }^{1,2}$, Jiufeng Sun ${ }^{1,2}$, Hengchang Sun ${ }^{1,2}$, Feifei Jia ${ }^{1,2}$, Chi Liang ${ }^{1,2}$, Xuerong Li $i^{1,2}$, Xiaonong Zhou ${ }^{3}$, Yan Huang ${ }^{1,2^{*}}$ and Xinbing $\mathrm{Yu}^{1,2^{*}}$

\begin{abstract}
Background: Clonorchis sinensis (C. sinensis, Cs) is a trematode parasite that often causes chronic cumulative infections in the hepatobiliary ducts of the host and can lead to pathological changes by continuously released excretory/secretory proteins (ESPs). A T2 ribonuclease in trematode ESPs, has been identified as a potent regulator of dendritic cell (DCs) modulation. We wondered whether there was a counterpart present in CsESPs with similar activity. To gain a better understanding of CSESPs associated immune responses, we identified and characterized RNASET2 of C. sinensis (CSRNASET2) in this paper.
\end{abstract}

Methods: We expressed CSRNASET2 in Pichia pastoris and identified its molecular characteristics using bioinformatic analysis and experimental approaches. The immune modulation activities of CSRNASET2 were confirmed by evaluating cytokine production and surface markers of recombinant CSRNASET2 (rCsRNASET2) co-cultured DCs, and monitoring levels of $\mathrm{lgG}$ isotypes from rCSRNASET2 administered BALB/c mice.

Results: CSRNASET2 appeared to be a glycoprotein of T2 ribonuclease family harboring conserved CAS motifs and rich in B-cell epitopes. Furthermore, CSRNASET2 was present in CSESPs and was able to modulate cytokine production of DCs. In addition, rCsRNASET2 could significantly suppress the expression of lipopolysaccharide-induced DCs maturation markers. In addition, when subcutaneously administered with rCSRNASET2 there was a marked effect on IgG isotypes in mouse sera.

Conclusion: Collectively, we revealed that CSRNASET2, a T2 ribonuclease present in CSESPs, could modulate DCs maturation and might play an important role in C. sinensis associated immune regulation in the host.

Keywords: Clonorchis sinensis, RNASET2, T2 ribonuclease, Excretory/secretory product, Dendritic cell, Immune modulation

\section{Background}

Clonorchiasis, caused by the infection of Clonorchis sinensis (C. sinensis), is highly epidemic in several Asian countries. More than 35 million people are infected with C. sinensis globally [1]. Clonorchiasis is predominantly caused by ingesting raw or undercooked freshwater fish harboring metacercariae [2]. When ingested by the host, larvae excyst in the duodenum and then migrate into

\footnotetext{
*Correspondence: huang66@mail.sysu.edu.cn; yuhxteam@163.com 'Department of Parasitology, Zhongshan School of Medicine, Sun Yat-sen University, Guangzhou 510080, People's Republic of China

${ }^{2}$ Key Laboratory for Tropical Diseases Control, Ministry of Education, Sun Yat-sen University, Guangzhou 510080, People's Republic of China Full list of author information is available at the end of the article
}

the peripheral intrahepatic bile ducts to develop as adult worms [3]. C. sinensis adult worms can thrive for more than 10 years in humans [4]. People infected with $C$. sinensis are often asymptomatic, however, repeated and chronic infections can eventually lead to various hepatobiliary symptoms and complications. During the long term of parasitism, the liver flukes continuously release excretory/secretory proteins (ESPs), a cocktail of hundreds to thousands of bioactive proteins. Prior studies have demonstrated that the components of ESPs from C. sinensis (CsESPs) are implicated in biological processes especially the induction of host immune response [5], which may be intimately associated with formation 
of cholangitis, liver cirrhosis and cholangiocarcinoma (CCA) [6]. Owing to its carcinogenicity, C. sinensis has been regarded as a group I carcinogen of cholangiocarcinoma [7].

T2 ribonucleases are transferase-type RNase and distribute broadly in almost all groups of living organisms including bacteria, fungi, virus, plants, and animals [8]. All of these enzymes have diverse biological activities, such as degradation of self-RNA, clearance of nucleic acids, serving as extra- or intracellular cytotoxins, and regulation of host immune responses [8-10]. Numerous studies have been conducted to characterize the biochemical properties and immunoregulatory roles of a T2 ribonuclease (omega1) in Schistosoma mansoni (Smomega-1), which is present in soluble egg antigens (SEA) of $S$. mansoni, formed by egg released ESPs [11-13]. Smomega-1 could trigger Th2 response by modulating dendritic cells (DCs) phenotype, structure and protein synthesis [14-16]. Moreover, Smomega-1 has been reported to induce priming of Foxp $3^{+}$ Tregs, which play crucial immunoregulatory roles in the process of $S$. mansoni infection [17]. Interestingly, the function of Smomega-1 to enhance Foxp3 expression also depends on the alteration of DCs. These findings illustrate that Smomega-1 is a key component of ESPs to modulate host immune responses, which is elicited by DCs alteration. DCs are defined as professional antigen-presenting cells serving as the sentinels of the immune system and have the unique capacity to induce and coordinate both innate and adaptive immune responses [18-22]. These findings give us insight into interactions between a secreted antigen and DCs, leading us to gain a better understanding of helminth associated immune responses.

In the present study, we expressed and characterized $C$. sinensis RNASET2 (CsRNASET2). We investigated the effects of rCsRNASET2 on bone marrow derived dendritic cell (BMDCs) modulation. In addition, we evaluated humoral immune responses initiated by $\mathrm{rCs}$ RNASET2 in $\mathrm{BALB} / \mathrm{c}$ mice. Based on these observations, we proposed that CsRNASET2 was intimately associated with C. sinensis-triggered immune response in the host.

\section{Methods}

\section{Animals}

Female 6- to 8-week-old BALB/c mice and SD rats were obtained from the animal center of Sun Yat-Sen University (Guangzhou, China). The animals were housed in a pathogen-free facility. All animal experiments were conducted under Animal Care and Use Committee of Sun Yat-sen University (Permit No: SCXK (Guangdong) 2009-0011).

\section{Sequence analysis of CSRNASET2}

The full-length encoding sequence of CsRNASET2 [Accession No. GAA50115.1] was downloaded from GenBank in NCBI [http://www.ncbi.nlm.nih.gov/]. Homology analysis was performed using the blastx program [http://blast.ncbi. nlm.nih.gov/]. The molecular characteristics and the functional domains of CsRNASET2 were assessed using proteomics tools provided by Expasy website [http://www.expasy. org/]. The alignments of the deduced amino acid sequence and T2 amino acid sequences from other species were performed by the software Vector NTI suite 8.0.

\section{Preparation of antigens and polyclonal antibodies}

Recombinant CsRNASET2 was expressed in $P$. pastoris according to the manufacturer's protocol (Invitrogen, USA). RNase activity of rCsRNASET2 was identified, and deglycosylation assay of the protein was carried out with PNGase F (New England BioLabs, USA). We collected CsESPs referring to the protocol as previously described [23]. Each BALB/c mouse was immunized subcutaneously with $100 \mu \mathrm{g}$ rCsRNASET2 or ESPs, which were respectively emulsified with complete Freund's adjuvant at the first injection. Two booster injections at 2-week intervals were performed with $50 \mu \mathrm{g}$ of proteins, which were emulsified with incomplete Freund's adjuvant. After 2 weeks of the final boosting, anti-sera were collected and antibody titers were determined by ELISA. All anti-sera samples were split and stored at $-80^{\circ} \mathrm{C}$.

\section{Western blotting}

rCsRNASET2 and CsESPs were subjected to $12 \%$ SDSPAGE, and subsequently electrotransferred onto the PVDF membranes (Millipore, USA) for $1 \mathrm{~h}$ at $100 \mathrm{~V}$. The membranes were then blocked with 5\% skimmed milk in PBS for $2 \mathrm{~h}$ at room temperature, and incubated with mycepitope monoclonal antibody (1:1000 dilutions), his-tag monoclonal antibody, mouse anti-rCsRNASET2 sera (1:2000 dilutions), mouse anti- ESPs sera, naïve mouse and $C$. sinensis-infected mouse sera (1:100 dilutions) respectively. The membranes were then incubated with peroxidase-conjugated goat anti-mouse IgG (Proteintech, USA, 1:10,000 dilutions) for $1 \mathrm{~h}$ at room temprature. The reactions were visualized by enhanced chemiluminescence (ECL) method.

\section{Generation and stimulation of bone marrow derived dendritic cells}

Femurs were obtained from BALB/c mice and the muscle tissues were removed with scissors. The bone marrow cells (BMCs) were isolated by flushing the bone marrow with chilled RPMI-1640 medium. Red blood cells were lysed with red blood cell lysing buffer (Sigma, USA). The remaining $\mathrm{BMCs}$ were washed twice and then resuspended into $2 \times 10^{6} / \mathrm{ml}$. BMCs were cultured in complete RPMI-1640 supplemented with $20 \mathrm{ng} / \mathrm{ml}$ mouse GM-CSF (R\&D Systems, USA) and $10 \mathrm{ng} / \mathrm{ml}$ mouse IL-4 (R\&D Systems). On days 3 and 5, fresh culture medium including the supplements were added. Immature BMDCs were 
harvested on day 7 and stimulated with or without different concentrations of rCsRNASET2 $(0.05-50 \mu \mathrm{g} / \mathrm{ml})$ or ESPs $(10-160 \mu \mathrm{g} / \mathrm{ml})$ in the presence of $1 \mu \mathrm{g} / \mathrm{ml}$ LPS (sigma) for 12, 24 and 48 h. Generally, Th1- and Th2-polarizing Ag stimulate DCs via discrete pathways, coupled with distinct modifications of the DC maturation. Therefore, we utilized rCsFABP, a protein has been reported to induce Th1 immune responses, as a control protein [24,25].

\section{Flow cytometry}

Surface markers on pretreated BMDCs were detected by FCM using the following mAbs: FITC labeled antiCD11c (eBioscience, USA), PE labeled anti-CD80, antiCD86, APC labeled anti-CD40 (BD PharMingen, USA). For staining, BMDCs were washed twice with PBS containing
0.1\% BSA and $0.05 \%$ sodium azide. The cells were thereafter incubated with the respective mAbs for $30 \mathrm{~min}$ at $4^{\circ} \mathrm{C}$ in the dark. Then, cells were washed twice and resuspended in PBS. Flow cytometry was performed on a Beckman Coulter Gallios cytometer and analyzed using the Kaluza software (Beckman Coulter, USA).

\section{ELISA}

Cell-free supernatants were harvested at different time points after stimulation, and levels of IL-12p70 and IL10 were assessed by commercially available ELISA kits (eBioscience) according to the manufacturer's protocol. Sera were collected from $\mathrm{rCsRNASET} 2$ immunized mice or PBS immunized mice at 4 and 6 weeks after primary injection. Levels of IgG1 and IgG2a in sera were evaluated

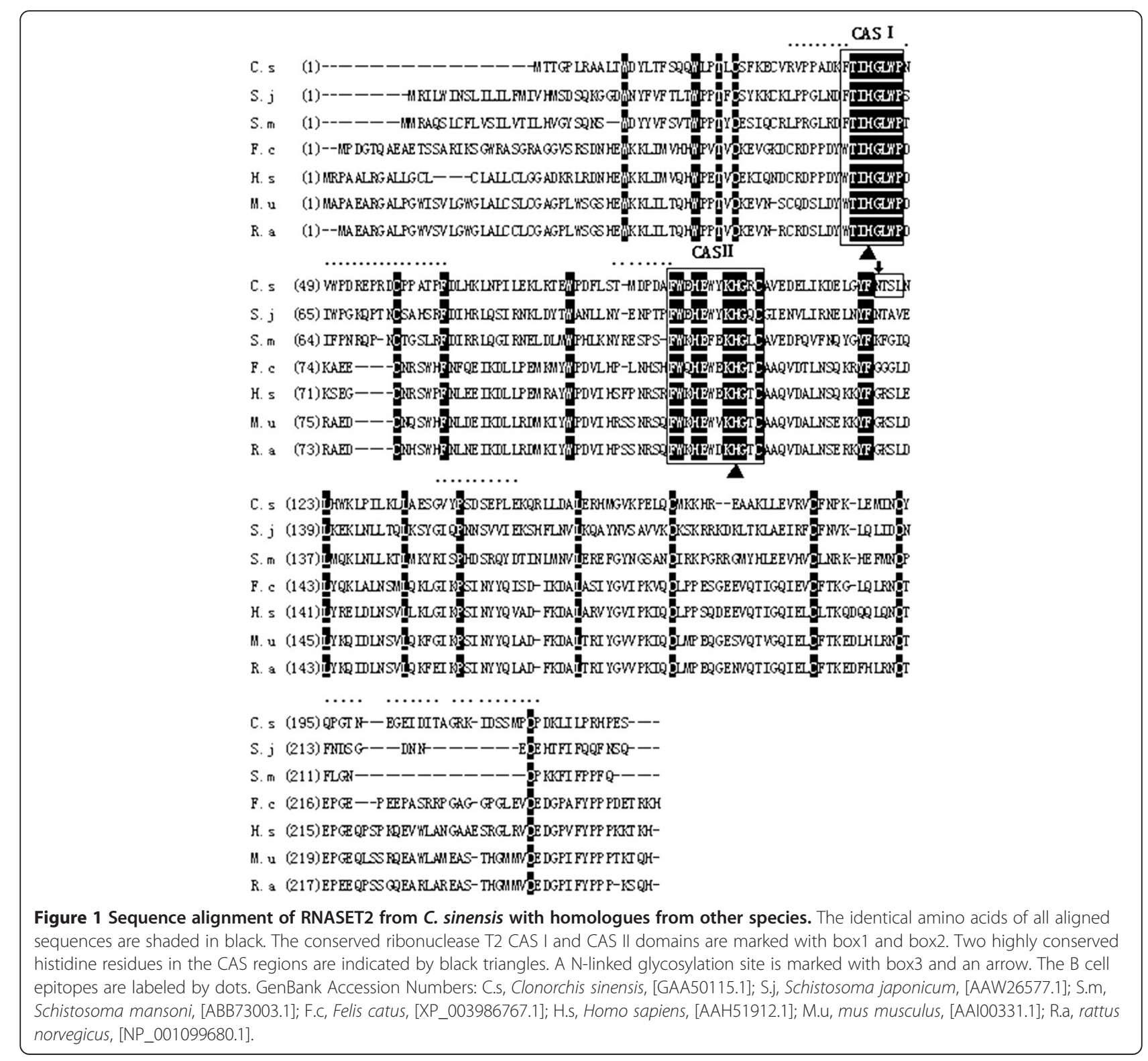


by ELISA coated with $100 \mu \mathrm{l} 5 \mu \mathrm{g} / \mathrm{ml} \mathrm{rCsRNASET} 2$ each well overnight at $4^{\circ} \mathrm{C}$. ELISA plates were blocked with $5 \%$ skimmed milk for $2 \mathrm{~h}$ at $37^{\circ} \mathrm{C}$ and then incubated with sera for $2 \mathrm{~h}$ at $37^{\circ} \mathrm{C}$. Subsequently, the plates were incubated with HRP-conjugated goat anti-mouse IgG1 and IgG2a (invitrogen) for $1 \mathrm{~h}$ at $37^{\circ} \mathrm{C}$. The optical density was tested at $450 \mathrm{~nm}$.

\section{Statistical analysis}

Data were routinely analyzed by the GraphPad Prism 5 software. The Mann-Whitney test was performed to calculate statistical significance of differences between two groups of observations. $p$-Values $<0.05$ were considered statistically significant.

\section{Results}

\section{Sequence analysis of CsRNASET2}

The complete coding sequence of CsRNASET2 contained 690 bp encoding a putative protein of 229 amino acids with the predicted molecular weight of $26.6 \mathrm{kDa}$. The hypothetical pI of the deduced protein was 5.65. The estimated half-life of CsRNASET2 was more than 20 hours in yeast and more than 10 hours in E. coli. The instability index of the deduced protein was 35.40 , indicating CsRNASET2 might be a stable protein. Blastx analysis showed that the sequence was a glycoprotein of the T2 ribonuclease family, which contained two critical histidine residues in the CAS motifs. However, the sequence just shared $43 \%$ and $39 \%$ identity with its homologues from S. janpani and S. mansoni, and it was rich in B-cell epitopes, which might suggest CsRNASET2 as being a good immunogen (Figure 1).

\section{Identification of CSRNASET2 as a component of C. Sinensis ESPs}

We successfully expressed $\mathrm{rCsRNASET} 2$ (Additional file 1: Figure $\mathrm{S} 1 \mathrm{~A}$ ) and identified its c-myc epitope and his-tag by western blot (Additional file 1: Figure S1B). The RNase activity of rCsRNASET2 was determined (Additional file 1: Figure S2). We noted that deglycosylated $\mathrm{r} C s$ RNASET2 displayed $\sim 4 \mathrm{kDa}$ reduction of molecular weight (Figure 2A). In addition, rCsRNASET2 could be probed by anti-CsRNASET2, anti-CsESPs and $C$. sinensis-infected mouse sera at a prominent single band around $35 \mathrm{kDa}$, whereas the protein could not be recognized by sera from naïve mice. In addition, $C s E S P s$ reacted with mouse anti-CsRNASET2 sera at a single band around $27 \mathrm{kDa}$ (Figure 2B). These results indicated that rCsRNASET2 was indeed a component of CsESPs with glycosylation and RNase activity.

\section{Cytokine production of BMDCs in response to rCsRNASET2} IL-12p70 and IL-10 are potent adjustive cytokines in the immune system, which can be secreted by DCs [26,27].

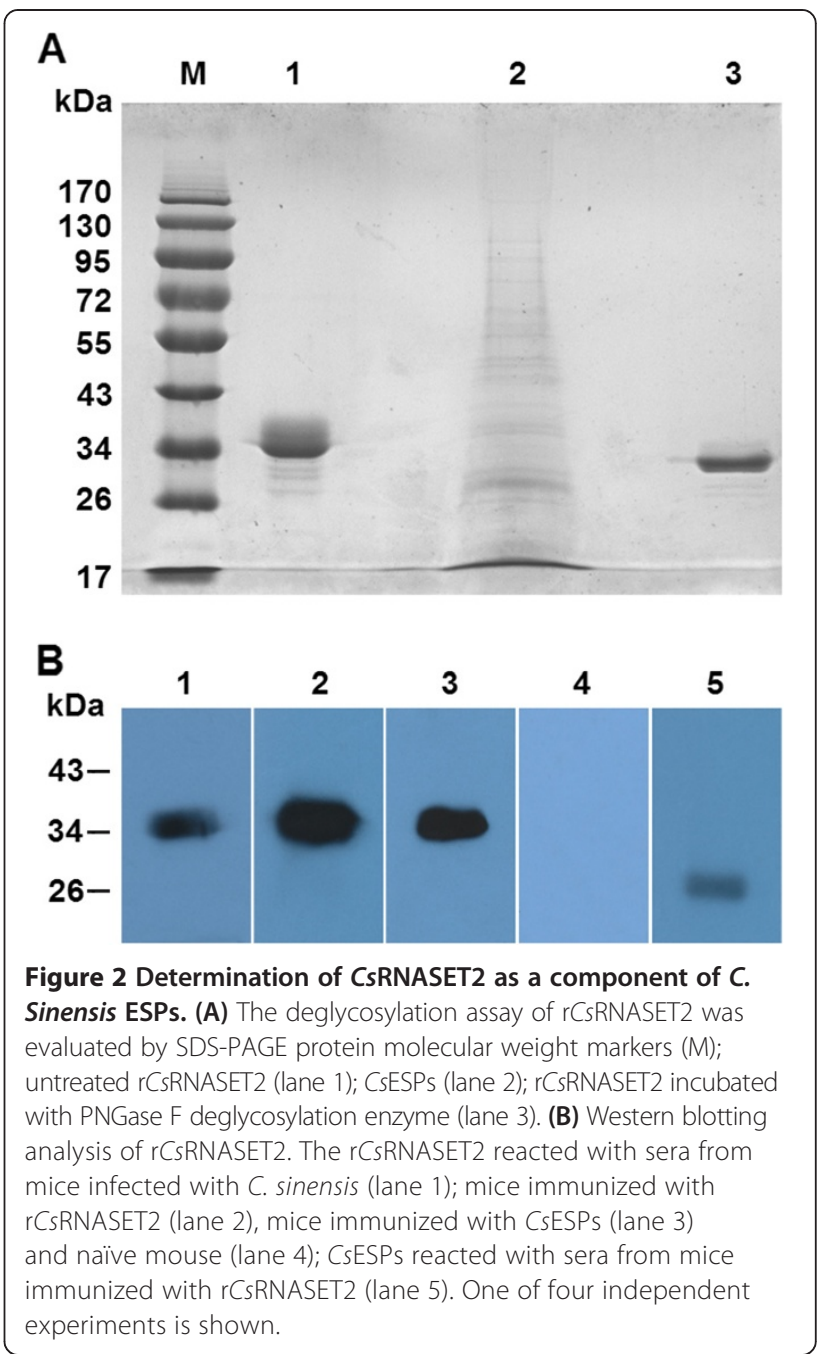

We incubated BMDCs with LPS in the presence or absence of various concentrations of rCsRNASET2, ESP or $0.5 \mu \mathrm{g} / \mathrm{ml} \mathrm{rCsFABP}$. We found that both rCsRNASET2 and CsESPs suppressed the LPS-induced up-regulation of IL-12p70 on BMDCs in a dose-dependent manner at the indicated time points (Figure 3A). In contrast, CsESPs (20$80 \mu \mathrm{g} / \mathrm{ml}$ ) enhanced the expression of IL-10 in a dosedependent manner, and rCsRNASET2 could promote IL-10 synthesis only at a concentration of $0.5 \mu \mathrm{g} / \mathrm{ml}$ (Figure 3B). In addition, rCsFABP could not obviously interfere with the cytokine production of DCs.

Effects of rCsRNASET2 on BMDCs phenotypic modifications A previous study has demonstrated that helminth antigens can affect the maturation of DCs [28]. To confirm the effects of CsRNASET2 on DCs maturation, BMDCs were treated with LPS in the presence or absence of rCsRNASET2, rCsFABP (control protein) or ESPs for $24 \mathrm{~h}$, and a series of surface markers expressed on DCs were assessed. 

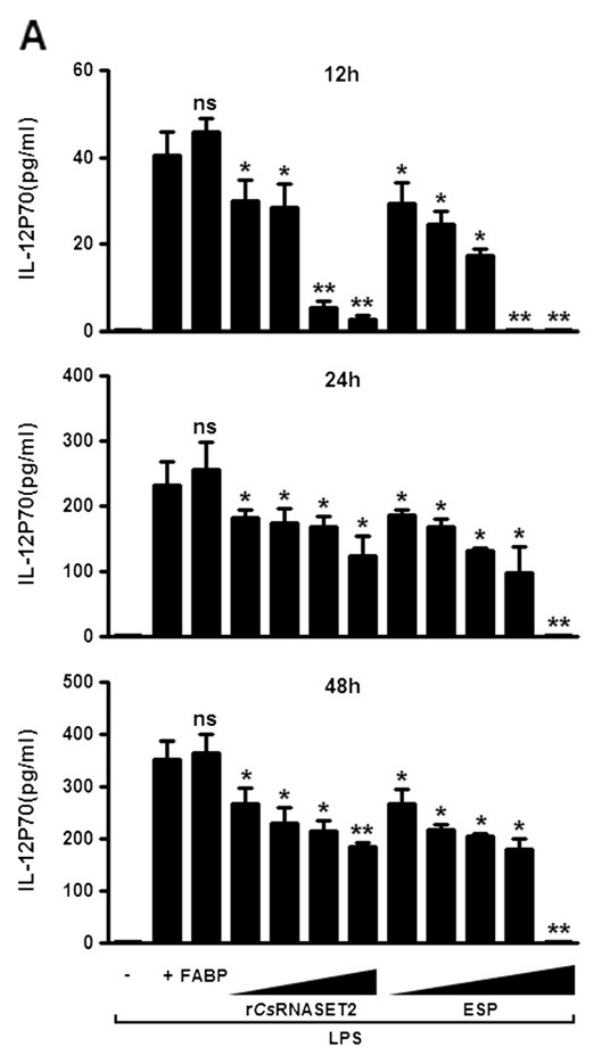

B
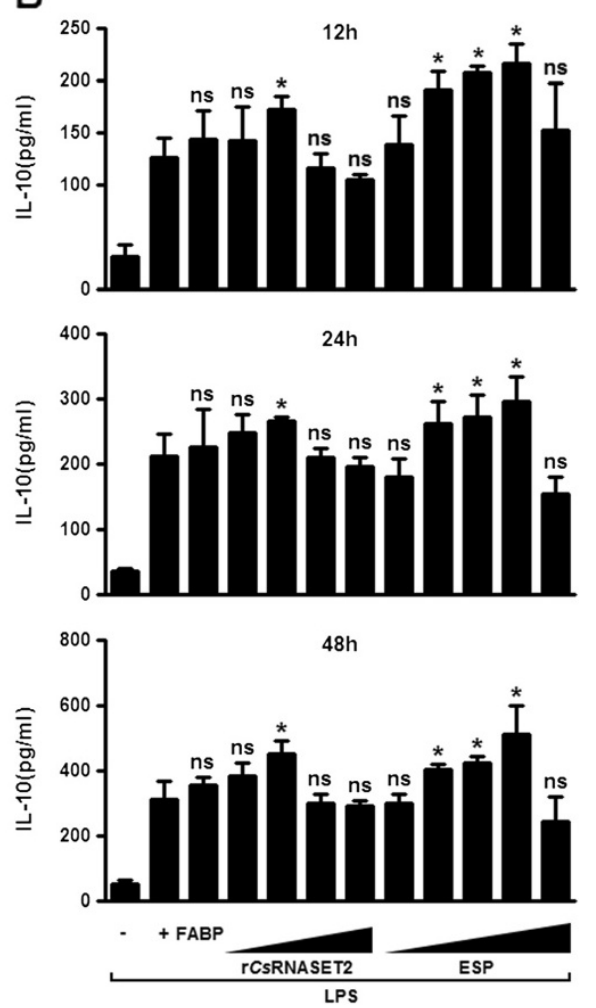

Figure 3 Cytokine production by BMDCs in response to rCsRNASET2. BMDCs $\left(2 \times 10^{6} / \mathrm{ml}\right)$ were incubated with $1 \mu \mathrm{g} / \mathrm{ml}$ LPS in the presence or absence of various concentrations of $(10,20,40,80$ and $160 \mu \mathrm{g} / \mathrm{ml})$ ESPs, $(0.05,0.5,5$ and $50 \mu \mathrm{g} / \mathrm{ml}) \mathrm{rCsRNASET} 2 \mathrm{or}(0.5 \mu \mathrm{g} / \mathrm{ml}) \mathrm{rCsFABP}$. Supernatants were harvested at different time points after stimulation and evaluated by ELISA for expression of IL-12p70 (A) and IL-10 (B). Error bars represent mean cytokine concentrations \pm SD $(n=5)$. Statistical significance was analyzed by the Mann-Whitney test $\left({ }^{*} p<0.05,{ }^{* *} p<0.01, n s\right.$ : not significant).

In the presence of LPS alone, the expression levels of CD11c, CD40, CD80 and CD86 on BMDCs were raised, when rCsRNASET2 or ESPs were added, the LPS-triggered up-regulation of surface markers was strikingly reduced. In contrast, rCsFABP had no significant effect on the expression of these surface markers (Figure 4A, B).

\section{IgG isotype analysis of rCsRNASET2 immunized mice}

For mice, the IgG1 response generally represents Th2activity, while IgG2a represents Th1-activity [29]. We evaluated the levels of IgG1 and IgG2a in sera obtained from $\mathrm{rCs}$ RNASET2 immunized mice. The data shown in Figure 5 shows that administration of $\mathrm{rCsRNASET} 2$ triggered a markedly higher IgG1 expression than IgG2a compared with control mice at week $4(1.99 \pm$ 0.12 versus $0.26 \pm 0.04, p<0.0001)$ and week $6(2.88 \pm$ 0.22 versus $0.49 \pm 0.31, p=0.0002$ ).

\section{Discussion}

It is well demonstrated that DCs are dominant players in the initiation and sustenance of immune responses [19-22]. In general, DCs undergo activation, termed maturation, upon recognition of invading pathogens, such as viruses, bacteria, and fungi. In the process of maturation, DCs up-regulate the production of surface markers and polarizing cytokines [20,21]. However, recent studies have indicated that DCs exposed to antigens derived from parasitic helminths fail to induce the classical DC activation [30-32]. The maturation status of DCs is crucial for the initiation of primary immune responses. It has been documented that Smomega-1, a RNase T2 family glycoprotein present in ESP, is a major molecule to dramatically modulate the maturation of DCs in S. mansoni infections [14-17].

Since Smomega-1 has been reported to play a potent role in the process of DCs modulation, we wondered whether there was a counterpart present in CsESPs with similar activity. In this study, we firstly identified a gene encoding CsRNASET2, which also appeared to be a glycoprotein of RNase T2 family. All RNase T2 enzymes have two blocks of conserved amino acids (termed CAS I and CAS II) $[8,9]$. These regions have typical catalytic residue of one to three histidine residues [8,33]. Sequence analysis showed that CsRNASET2 indeed harbored the two 


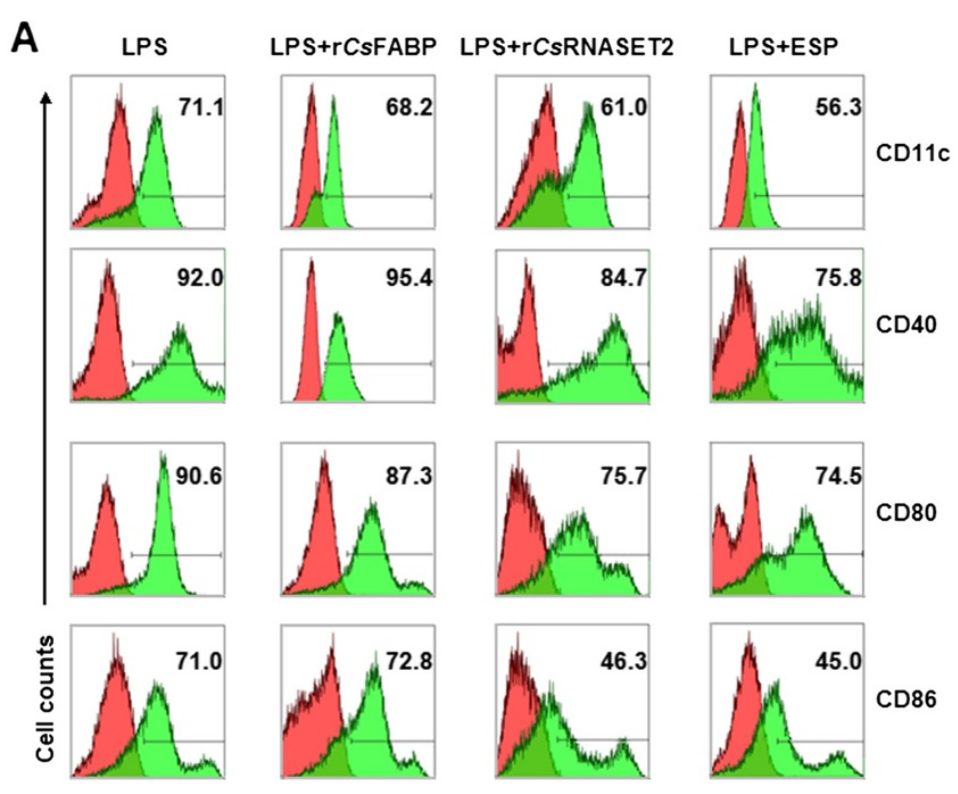

\section{B}

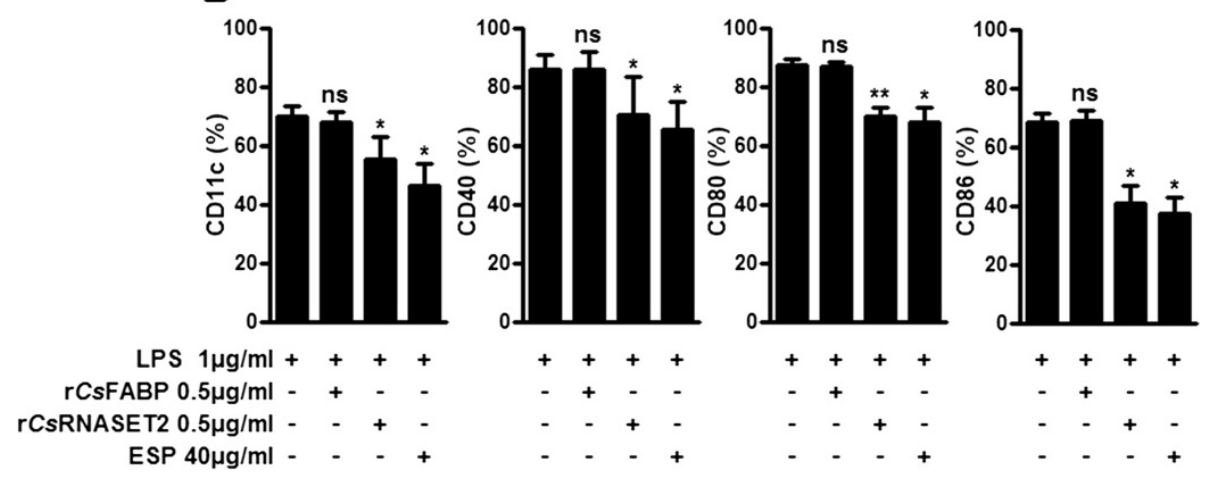

Figure 4 Modulation of BMDCs maturation elicited by rCsRNASET2. (A) Days-7 BMDCs $\left(2 \times 10^{6} / \mathrm{ml}\right)$ were stimulated with or without $0.5 \mu \mathrm{g} /$ $\mathrm{ml} \mathrm{rCSRNASET2,} 0.5 \mu \mathrm{g} / \mathrm{ml} \mathrm{rCSFABP}$ or $40 \mu \mathrm{g} / \mathrm{ml}$ ESPs in the presence of $1 \mu \mathrm{g} / \mathrm{ml}$ LPS for $24 \mathrm{~h}$. The levels of CD11c, CD40, CD80 and CD86 on BMDCs were detected by flow cytometry. Values stand for the percentage of treated cells staining positive for the indicated marker. Histograms are representative of seven independent experiments. (B) Statistical results of the expression of surface markers on BMDCs. Data are expressed as mean \pm SD. Statistical significance was analyzed by the Mann-Whitney test $\left({ }^{*} p<0.05,{ }^{* *} p<0.01\right.$, ns: not significant).

conserved CAS I and CAS II regions with two critical histidine residues (Figure 1). It suggested that CsRNASET2 was the counterpart of Smomega-1. We expressed rCsRNASET2 in P. pastoris with RNase activity. Deglycosylation experiment displayed $\sim 4 \mathrm{kDa}$ size reduction of the rCsRNASET2 (Figure 2A), which indicated the rCsRNASET2 was indeed glycosylated. rCsRNASET2 could react with $C$. sinensis-infected mouse sera and ESPs immunized mouse sera, meanwhile, ESPs could be probed with mouse anti-rCsRNASET2 sera at a single band (Figure 2B). These results revealed that CsRNASET2 was indeed a component of CsESPs, which are in accordance with the fact that the RNase T2 family members are typically secreted $[9,34]$. The difference of $8 \mathrm{kDa}$ in molecular weight between rCsRNASET2 and the native protein in ESP might be due to his/ myc-tag, a minor imprecision on SDS-PAGE assays and glycosylation ( $\sim 4 \mathrm{kDa}$ reduction). Meanwhile, we performed western blot analysis, in which ESP reacted with anti-rCsRNASET2 and anti-ESP mouse sera. Gradation analysis revealed that CsRNASET2 took up about $2 \%$ of ESP (Additional file 1: Figure S3).

Furthermore, ELISA assays revealed that the LPSinduced up-regulation of IL-12p70 on BMDCs decreased in a dose-dependent manner in the presence of various concentrations of rCsRNASET2 or CsESP (Figure 3A). In contrast, CsESPs $(20-80 \mu \mathrm{g} / \mathrm{ml})$ enhanced the expression of IL-10 in a dose-dependent manner, and rCsRNASET2 could promote IL-10 synthesis only at a concentration of $0.5 \mu \mathrm{g} / \mathrm{ml}$. Higher concentrations of rCsRNASET2 or ESP failed to promote IL-10 production (Figure $3 \mathrm{~B}$ ). We then performed BMDCs survival assay with Cell Counting kit-8 (CCK8) under different concentrations of rCsRNASET2 

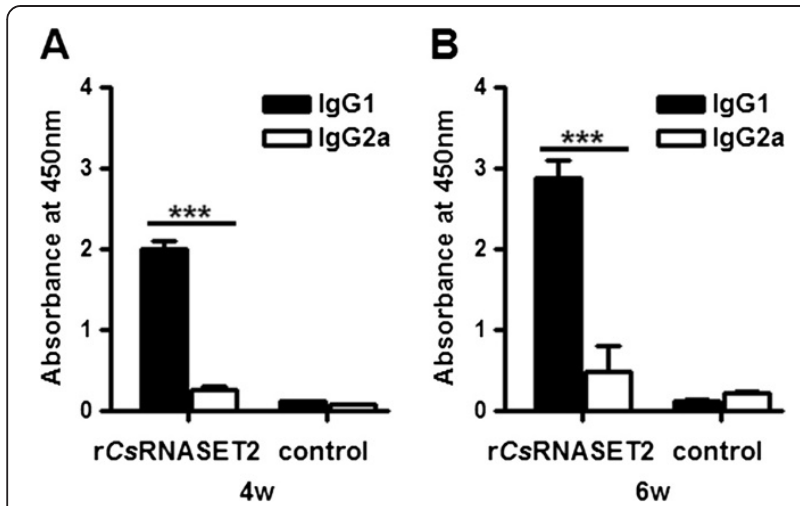

Figure 5 IgG subclass responses triggered by rCSRNASET2. BALB/c mice $(n=6)$ were immunized s.c. with rCSRNASET2. After 4 (A) and 6 (B) weeks, sera levels of lgG1 and lgG2a specific for rCSRNASET2 were evaluated by ELISA. IgG1 levels were significantly higher than $\lg \mathrm{G} 2 \mathrm{a}$ at both 4th and 6th week. Error bars represent mean levels \pm SD. Statistical significance was analyzed by the MannWhitney test $(* * * 0.0005)$.

or ESP, and observed that higher concentrations of rCsRNASET2 or ESP could inhibit cell viability (Additional file 1: Figure S4). Therefore, we finally chose $0.5 \mu \mathrm{g} / \mathrm{ml}$ of rCsRNASET2 and $40 \mu \mathrm{g} / \mathrm{ml}$ of ESP as moderate working concentrations. CsFABP, a Th1-polarizing Ag, was utilized to be a control protein. There was no significant difference of cytokine production levels when $\mathrm{rCsFABP}$ was added. Subsequently, we carried out flow cytometry analysis and noted that levels of surface markers including CD11C, CD40, CD80 and CD86 on rCsRNASET2- or CsESPtreated BMDCs significantly decreased compared with LPS controls, whereas rCsFABP displayed little effect on these surface makers expression (Figure 4). These results demonstrated that rCsRNASET2 could modulate the cytokine production and maturation of DCs. Interleukin-12 (IL-12) plays the key role in the generation of Th1 cells. IL-12p70 is a heterodimeric cytokine composed of two chains (p35 and p40), which is able to stimulate NK cells and T cells to produce IFN- $\gamma$ to resist against pathogens [35]. It has been well established that IL-10 is a cytokine secreted by $\mathrm{CD} 4^{+} \mathrm{T}$ cells belonging to the Th2 subset, which has been determined to suppress the production of IFN- $\gamma$. The induction of IL-10 may be an important strategy by which parasites evade IFN- $\gamma$-dependent, cell-mediated immune destruction [36]. Our experiments showed diminished expression of IL-12p70 and increased expression of IL-10 in rCsRNASET2-cocultured DCs. Therefore, it is conceivable that rCsRNASET2 co-cultured DCs may suppress Th1 polarization, which is eventually beneficial for C. sinensis escaping from the host immunity.

IgG2a and IgGl immunoglobulin isotypes are generally regarded as markers for Th1 and Th2 responses respectively [29,37]. Our studies demonstrated that the upregulation of $\operatorname{IgG1}$ was greater than that of $\operatorname{IgG2a}$ (Figure 5), confirming that Th2 responses might be dominant in rCsRNASET2 immunized mice. These results were in agreement with the data obtained from rCsRNASET2 coincubated BMDCs, as reduced expression of IL-12p70 and increased production of IL-10 could inhibit Th1 polarization. However, it is difficult to determine the dominance of Th1 or Th2 responses on the basis of IgG isotypes alone, because the initiation of $\mathrm{B}$ cell isotype switching is not only confined to the mutual effects of Th1 and Th2 cytokines. Further studies are required to determine whether CsRNASET2 is able to trigger Th2 or Treg responses through DCs modulation, whether this function is associated with its RNase activity, and which pathways are involved in the process.

\section{Conclusion}

Collectively, we cloned and identified CsRNASET2 as a molecule present in C. sinensis ESPs. We confirmed that rCsRNASET2 could regulate the host immune response via modulating DCs maturation, as well as production of IL-12p70 and IL-10. We also observed that administration of rCsRNASET2 to mice could alter the IgG1/IgG2a ratio. It will be of significant interest to investigate whether CsRNASET2 is able to initiate Th2 or Treg responses through modulating DCs, which will pave the way towards unraveling the regulatory mechanisms of the immune responses in C. sinensis infection and may provide potential therapeutic tools.

\section{Additional file}

Additional file 1: Figure S1. Expression and purification of rCSRNASET2. Figure S2. Determination of ribonuclease activity of rCSRNASET2. Figure S3. Relative quantification of CSRNASET2 in CSESP. Figure S4. Cytotoxity assessment of rCSRNASET2.

\section{Competing interests}

The authors declare that they have no competing interests.

\section{Authors' contributions}

YQX, XNZ, YH and XBY conceived and designed the experiments; YQX, WJC, $M B, X Y W$, JFS, HCS, FFJ, CL, XRL performed the experiments; YQX, WJC and MB analyzed the data; YQX, WJC, XYW and YH wrote the manuscript; All authors read and approved the final manuscript.

\section{Acknowledgments}

This work was supported by grants from the National Key Basic Research and Development Project of China (973 project; No.2010CB530000), the National Important Sci-tech Special Projects (No. 2012ZX10004220), National Natural Science Foundation of China (No. 81101270 and No. 81171602), the Fundamental Research Funds for the Central Universities (No. 3164015) and Sun Yat-sen University graduate student funding for abroad visiting and international cooperation research projects.

\section{Author details}

'Department of Parasitology, Zhongshan School of Medicine, Sun Yat-sen University, Guangzhou 510080, People's Republic of China. ${ }^{2}$ Key Laboratory for Tropical Diseases Control, Ministry of Education, Sun Yat-sen University, Guangzhou 510080, People's Republic of China. ${ }^{3}$ National Institute of 
Parasitic Diseases, Chinese Center for Disease Control and Prevention, Shanghai 200025, People's Republic of China.

Received: 4 September 2013 Accepted: 18 December 2013 Published: 23 December 2013

\section{References}

1. Lin RQ, Tang JD, Zhou DH, Song HQ, Huang SY, Chen JX, Chen MX, Zhang $H$, Zhu XQ, Zhou XN: Prevalence of Clonorchis sinensis infection in dogs and cats in subtropical southern China. Parasit Vectors 2011, 4:180.

2. Chen J, Xu MJ, Zhou DH, Song HQ, Wang CR, Zhu XQ: Canine and feline parasitic zoonoses in China. Parasit Vectors 2012, 5:152.

3. Kim HG, Han J, Kim MH, Cho KH, Shin IH, Kim GH, Kim JS, Kim JB, Kim TN, Kim TH, Kim TH, Kim JW, Ryu JK, Moon YS, Moon JH, Park SJ, Park CG, Bang SJ, Yang CH, Yoo KS, Yoo BM, Lee KT, Lee DK, Lee BS, Lee SS, Lee SO, Lee WJ, Cho CM, Joo YE, Cheon GJ, et al: Prevalence of clonorchiasis in patients with gastrointestinal disease: a Korean nationwide multicenter survey. World J Gastroenterol 2009, 15:86-94.

4. Keiser J, Utzinger J: Food-borne trematodiases. Clin Microbiol Rev 2009, 22:466-483.

5. Lei HL, Tian YL, Chen WJ, Wang XY, Li XR, Mao Q, Sun JF, Li R, Xu YQ, Liang C, Huang $Y, Y u X B$ : The biochemical and immunological characterization of two serpins from Clonorchis sinensis. Mol Biol Rep 2013, 40:3977-3985.

6. Qian MB, Chen YD, Fang YY, Xu LQ, Zhu TJ, Tan T, Zhou CH, Wang GF, Jia TW, Yang GJ, Zhou XN: Disability weight of Clonorchis sinensis infection: captured from community study and model simulation. PLOS Negl Trop Dis 2011, 5:e1377.

7. Fried B, Reddy A, Mayer D: Helminths in human carcinogenesis. Cancer Lett 2011, 305:239-249.

8. Luhtala N, Parker R: T2 Family ribonucleases: ancient enzymes with diverse roles. Trends Biochem Sci 2010, 35:253-259.

9. Deshpande RA, Shankar V: Ribonucleases from T2 family. Crit Rev Microbiol 2002, 28:79-122.

10. Schwartz B, Shoseyov O, Melnikova VO, McCarty M, Leslie M, Roiz L, Smirnoff P, Hu GF, Lev D, Bar-Eli M: ACTIBIND, a T2 RNase, competes with angiogenin and inhibits human melanoma growth, angiogenesis and metastasis. Cancer Res 2007, 67:5258-5266.

11. Dunne DW, Jones FM, Doenhoff MJ: The purification, characterization, serological activity and hepatotoxic properties of two cationic glycoproteins (alpha 1 and omega 1) from Schistosoma mansoni eggs. Parasitology 1991, 103:225-236.

12. Fitzsimmons CM, Schramm G, Jones FM, Chalmers IW, Hoffmann KF, Grevelding CG, Wuhrer M, Hokke CH, Haas H, Doenhoff MJ, Dunne DW: Molecular characterization of omega-1: a hepatotoxic ribonuclease from Schistosoma mansoni eggs. Mol Biochem Parasitol 2005, 144:123-127.

13. Meevissen MH, Wuhrer M, Doenhoff MJ, Schramm G, Haas H, Deelder AM, Hokke $\mathrm{CH}$ : Structural characterization of glycans on omega-1, a major Schistosoma mansoni egg glycoprotein that drives Th2 responses. J Proteome Res 2010, 9:2630-2642.

14. Everts $B$, Hussaarts $L$, Driessen NN, Meevissen $M H$, Schramm G, van der Ham AJ, van der Hoeven B, Scholzen T, Burgdorf S, Mohrs M, Pearce EJ, Hokke $\mathrm{CH}$, Haas $\mathrm{H}$, Smits HH, Yazdanbakhsh M: Schistosome-derived omega-1 drives Th2 polarization by suppressing protein synthesis following internalization by the mannose receptor. J Exp Med 2012, 209:1753-1767.

15. Everts B, Perona-Wright G, Smits HH, Hokke CH, van der Ham AJ, Fitzsimmons CM, Doenhoff MJ, van der Bosch J, Mohrs K, Haas H, Mohrs M, Yazdanbakhsh M, Schramm G: Omega-1, a glycoprotein secreted by Schistosoma mansoni eggs, drives Th2 responses. J Exp Med 2009, 206:1673-1680.

16. Steinfelder S, Andersen JF, Cannons JL, Feng CG, Joshi M, Dwyer D, Caspar P, Schwartzberg PL, Sher A, Jankovic D: The major component in schistosome eggs responsible for conditioning dendritic cells for Th2 polarization is a T2 ribonuclease (omega-1). J Exp Med 2009, 206:1681-1690.

17. Zaccone P, Burton OT, Gibbs SE, Miller N, Jones FM, Schramm G, Haas H, Doenhoff MJ, Dunne DW, Cooke A: The S. mansoni glycoprotein $\omega-1$ induces Foxp3 expression in NOD mouse $\mathrm{CD}^{+} \mathrm{T}$ cells. Eur J Immunol 2011, 41:2709-2718.

18. Banchereau J, Steinman RM: Dendritic cells and the control of immunity. Nature 1998, 392:245-252.

19. Steinman RW, Hawiger D, Nussenzweig MC: Tolerogenic dendritic cells. Annu Rev Immunol 2003, 21:685-711.
20. Banchereau J, Briere F, Caux C, Davoust J, Lebecque S, Liu YJ, Pulendran B, Palucka K: Immunobiology of dendritic cells. Annu Rev Immunol 2000, 18:767-811

21. Lipscomb MF, Masten BJ: Dendritic cells: immune regulators in health and disease. Physiol Rev 2002, 82:97-130.

22. Morelli AE, Thomson AW: Tolerogenic dendritic cells and the quest for transplant tolerance. Nat Rev Immunol 2007, 7:610-621.

23. Chen WJ, Wang XY, Li XR, Lv XL, Zhou CH, Deng CH, Lei HL, Men JT, Fan YX, Lian C, Yu XB: Molecular characterization of cathepsin B from Clonorchis sinensis excretory/secretory products and assessment of its potential for serodiagnosis of clonorchiasis. Parasit Vectors 2011, 4:149.

24. Cervi L, MacDonald AS, Kane C, Dzierszinski F, Pearce EJ: Cutting edge: dendritic cells copulsed with microbial and helminth antigens undergo modified maturation, segregate the antigens to distinct intracellular compartments, and concurrently induce microbe-specific Th1 and helminth-specific Th2 responses. J Immunol 2004, 172:2016-2020.

25. Lee JS, Kim IS, Sohn WM, Lee J, Yong TS: A DNA Vaccine Encoding a Fatty Acid-Binding Protein of Clonorchis sinensis Induces Protective Immune Response in Sprague-Dawley Rats. Scand J Immunol 2006, 63:169-176.

26. Trinchieri G, Pflanz S, Kastelein RA: The IL-12 family of heterodimeric cytokines: new players in the regulation of $\mathrm{T}$ cell responses. Immunity 2003, 19:641-644.

27. Moore KW, de Waal MR, Coffman RL, O'Garra A: Interleukin-10 and the interleukin-10 receptor. Annu Rev Immunol 2001, 19:683-765.

28. Everts B, Smits HH, Hokke CH, Yazdanbakhsh M: Helminths and dendritic cells: sensing and regulating via pattern recognition receptors, Th2 and Treg responses. Eur J Immunol 2010, 40:1525-1537.

29. Mountford AP, Fisher A, Wilson RA: The profile of IgGl and IgG2a antibody responses in mice exposed to Schistosoma mansoni. Parasite Immunol 1994, 16:521-527.

30. Jeong YI, Kim SH, Ju JW, Cho SH, Lee WJ, Park JW, Park YM, Lee SE: Clonorchis sinensis-derived total protein attenuates airway inflammation in murine asthma model by inducing regulatory $T$ cells and modulating dendritic cell functions. Biochem Biophys Res Commun 2011, 407:793-800.

31. MacDonald AS, Straw AD, Bauman B, Pearce EJ: CD8 ${ }^{-}$dendritic cell activation status plays an integral role in influencing Th2 response development. J Immunol 2001, 167:1982-1988.

32. MacDonald AS, Straw AD, Dalton NM, Pearce EJ: Cutting edge: Th2 response induction by dendritic cells: a role for CD40.J Immunol 2002, 168:537-540.

33. Kawata Y, Sakiyama F, Hayashi F, Kyogoku Y: Identification of two essential histidine residues of ribonuclease T2 from Aspergillus oryzae. Eur J Biochem 1990, 187:255-262.

34. Campomenosi P, Salis S, Lindqvist C, Mariani D, Nordström T, Acquati F, Taramelli R: Characterization of RNASET2, the first human member of the $\mathrm{Rh} / \mathrm{T} 2 / \mathrm{S}$ family of glycoproteins. Arch Biochem Biophys 2006, 449:17-26.

35. Hunter CA: New IL-12-family members: IL-23 and IL-27, cytokines with divergent functions. Nat Rev Immunol 2005, 5:521-531.

36. Ouyang W, Rutz S, Crellin NK, Valdez PA, Hymowitz SG: Regulation and functions of the IL-10 family of cytokines in inflammation and disease. Annu Rev Immunol 2011, 29:71-109.

37. Maassen CBM, Boersma WJA, van Holten-Neelen C, Claassen E, Laman JD: Growth phase of orally administered Lactobacillus strains differentially affects lgG1/lgG2a ratio for soluble antigens: implications for vaccine development. Vaccine 2003, 21:2751-2757.

doi:10.1186/1756-3305-6-360

Cite this article as: Xu et al.: Molecular characterization and immune modulation properties of Clonorchis sinensis-derived RNASET2. Parasites \& Vectors 2013 6:360 